\title{
Exogenous lipoid pneumonia induced by nasal instillation of paraffin oil
}

\begin{abstract}
Lipoid pneumonia is a rare pulmonary disease, classified in terms of the source of lipid exposure into two variants: exogenous and endogenous. We present a patient with exogenous lipoid pneumonia, acquired after chronic exposure to paraffin oil-containing nasal drops. The diagnosis was established by demonstration of lipid-laden macrophages in bronchoalveolar lavage, chest computed tomography results and a history of lipid exposure.
\end{abstract}

Key words: lipoid pneumonia; exogenous; foamy macrophages

Adv Respir Med. 2019; 87: 254-257

\section{Introduction}

Lipoid pneumonia is an uncommon condition, initially described in 1925 by Laughlen in autopsy series of three children and one adult $[1,2]$. Its clinical incidence is difficult to precise, however, one American autopsy study has reported the frequency of 1.0-2.5\% [1, 3, 4]. Exogenous lipoid pneumonia may occur in subjects of any age, however, it is more likely to affect the elderly, particularly those who have a history of chronic laxatives or nasal drop use.

The condition results from accumulations of lipids in the alveoli. Various clinical manifestations have been described. In about half of the cases, the disease is asymptomatic, in others, it manifests with cough and progressive dyspnoea on exertion. In patients with massive aspiration of lipids in short time, the symptoms of severe, life-threatening respiratory failure requiring ventilator support may develop [5].

Radiological presentations are diverse, there is no specific, pathognomonic finding, however, chest computed tomography (chest CT) plays a complementary role in the diagnosis, which is often difficult [1]. Therefore, a history of expo- sure should be investigated in details in order to confirm the diagnosis.

We report a case of a female patient with chronic exogenous lipoid pneumonia, which was diagnosed in the I Department of Lung Diseases, National Tuberculosis and Lung Diseases Research Institute.

\section{Case report}

A 57-year-old woman, active smoker, with a history of 30 pack-years, was admitted to our clinic because of progressive dyspnoea on exertion and dry cough, since the beginning of 2018. In February and August 2018, the patient suffered from infectious pneumonia, which has been successfully treated with antibiotics. Past medical history revealed chronic kidney disease (Stage G3a), diabetes mellitus type 2, arterial hypertension and duodenal ulcerations. Due to persistent opacities on the chest radiograph, a chest-CT has been performed, which showed multiple confluent areas of ground-glass opacities in the middle lobe, both lower lobes and areas of parenchymal consolidations with density similar to fat (between -150 and -30 Hounsfield units) in 


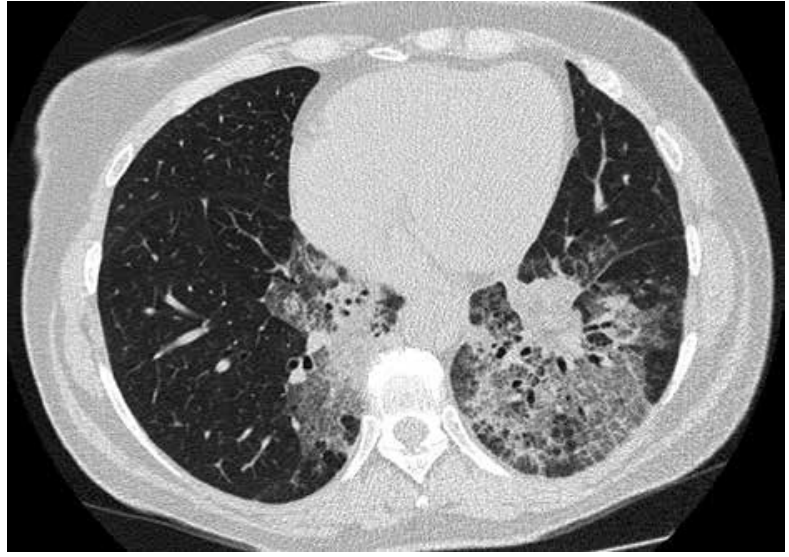

Figure 1. Chest CT scan at the level of lower lobes demonstrates areas of parenchymal consolidations especially in the left lung

segment 10 of the right lobe and basal segments of the left lower lobe (Figures 1 and 2).

At admission, oxygen saturation in room air was lowered to $88 \%$, heart rate was $62 \mathrm{bpm}$, blood pressure - 135/80 mm Hg. Auscultation of the lungs revealed bilateral basal crepitations. The blood tests showed no elevation of the inflammatory markers, a slightly elongated aPTT $(37,5 \mathrm{~s})$ and elevated D-dimer (1391 ng/mL) were noted. Antiphospholipid syndrome and other systemic connective tissue diseases were excluded (anti -nuclear antibodies like anti-dsDNA, anti-SS-A, anti-SS-B, Anti-RNP, anti-Scl-70, anti-Jo-1, antiMi-2, lupus anticoagulant and anticardiolipin antibodies were negative). Chest radiograph showed bilateral opacities, especially in the left supradiaphragmatic area (Figure 3). Lung function tests were normal (Table 1). The patient underwent a fiberoptic bronchoscopy, which did not reveal any pathologies in the main or segmental bronchi. Cultures of bronchial washings were negative. Bronchoalveolar lavage (BAL) from the left lower lobe was performed with $60 \%$ of recovery. Under macroscopic evaluation, the fluid was described as turbid, with fat-like layer on the surface. Cytological examination showed a significant amount of foamy macrophages, probably with accumulations of lipid material (Table 2 ). The specific probe staining was not performed due to technical reasons.

Based on BAL result, a carefully performed anamnesis revealed an over 10 years long exposure to paraffin oil-containing nasal drops, which patient was using due to nasal blockade congestion. Deviated nasal septum as a cause was confirmed in rhinoscopy. The patient was strongly advised to stop using the nasal drops. After three months on a control visit, clinically she was

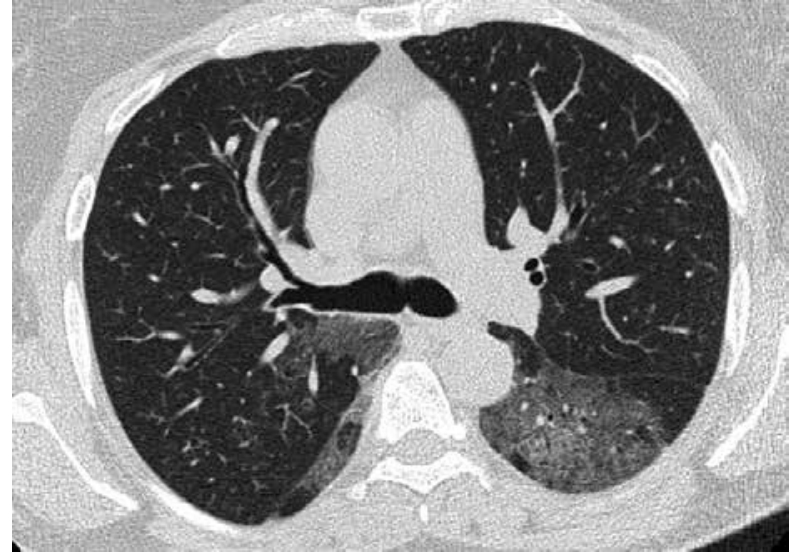

Figure 2. Chest CT scan at the level of middle lobe demonstrates areas of ground-glass attenuation

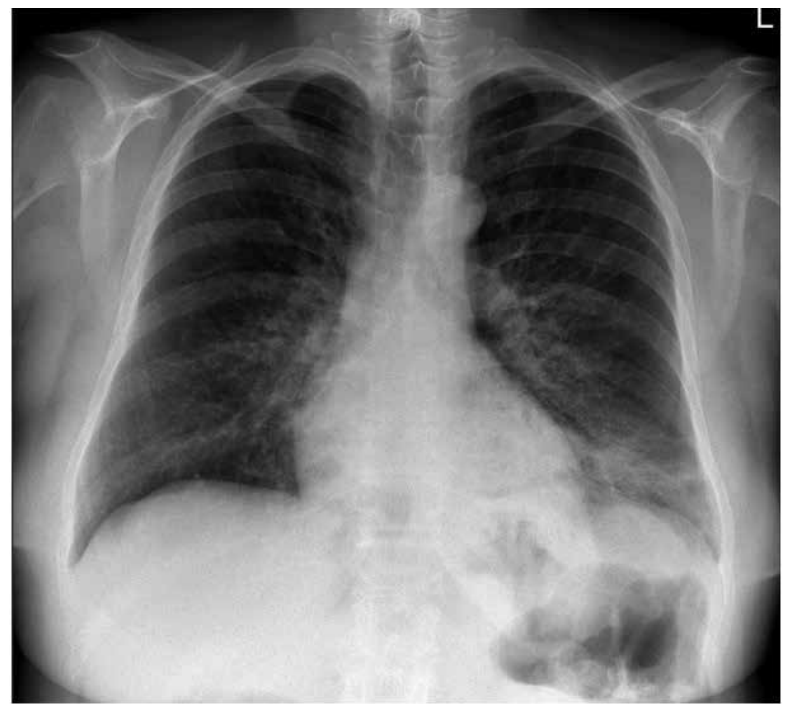

Figure 3. Chest radiograph shows bilateral opacities, especially in the left lung supradiaphragmatic area

well, lung function test was stable, although the radiological changes were still remaining.

\section{Discussion}

Lipoid pneumonia is a rare, nonspecific cause of inflammatory lung disease $[1,3,4,6]$.

Basing on the source of the lipids exposure, lipoid pneumonia has been classified into endogenous and exogenous [1]. The pathogenesis of the endogenous form is complex, the source of lipids is the lung tissue itself, the disease may occur as a result of infection (mainly in the obstructed bronchus), repetitive episodes of fungal infections or due to the non-infectious process such as pulmonary alveolar proteinosis and lipid-storage disorders (Niemann-Pick disease) [1, 3, 6, 7]. 
Table 1. Results of body plethysmography

\begin{tabular}{|c|c|c|c|c|c|c|}
\hline & Pred & Pre & $\%($ Pre/Pred) & Z-Score & Pred LL & Pred UL \\
\hline FEV1\% VC MAX (\%) & 78.27 & 81.22 & 104 & 0.45 & 67.56 & 88.98 \\
\hline FEV1\% FVC (\%) & 78.27 & 85.00 & 109 & 1.03 & 67.56 & 88.98 \\
\hline VC MAX (L) & 3.09 & 2.87 & 93 & -0.48 & 2.36 & 3.85 \\
\hline FVC (L) & 3.09 & 2.74 & 89 & -0.77 & 2.36 & 3.85 \\
\hline FEV1 (L) & 2.45 & 2.33 & 95 & -0.35 & 1.87 & 3.01 \\
\hline MMEF 75-25 (L/s) & 2.32 & 2.99 & 129 & 0.81 & 1.22 & 3.77 \\
\hline PEF (L/s) & 5.93 & 9.20 & 155 & 3.64 & 4.44 & 7.41 \\
\hline $\mathrm{R}$ tot $(\mathrm{kPA}(\mathrm{L} / \mathrm{s})$ & 0.30 & 0.36 & 120 & & 0.33 & 0.30 \\
\hline FRCpleth (L) & 2.62 & 2.46 & 94 & -0.32 & 1.80 & 3.44 \\
\hline $\mathrm{RV}(\mathrm{L})$ & 1.79 & 1.56 & 87 & -0.65 & 1.21 & 2.37 \\
\hline TLC (L) & 4.70 & 4.44 & 94 & -0.45 & 3.72 & 5.69 \\
\hline RV \% TLC (\%) & 38.34 & 35.26 & 92 & -0.53 & 28.75 & 47.93 \\
\hline $\begin{array}{l}\text { DLCOc single breath } \\
{[\mathrm{mmoL}(\mathrm{min} \times \mathrm{kPa})]}\end{array}$ & 6.53 & 4.74 & 73 & -1.97 & 4.98 & 8.72 \\
\hline $\begin{array}{l}\text { DLCOc_SB/NA [mmoL } \\
(\min \times \mathrm{kPa} \times \mathrm{L})]\end{array}$ & 1.45 & 1.15 & 79 & -1.48 & 1.12 & 1.83 \\
\hline VIN single breath (L) & 2.76 & 2.96 & 107 & 0.48 & 2.07 & 3.45 \\
\hline VA single breath (L) & 4.55 & 4.12 & 91 & -0.77 & 3.67 & 5.61 \\
\hline
\end{tabular}

DLCOc — diffusion lung capacity for carbon monoxide corrected value; FEV1 — forced expiratory volume in 1 second; FRC — functional residual capacity; FVC — forced vital capacity; KPA — kilopascal; L — liter; LL — lower line; MMEF — maximal expiratory flow; min — minute; $\mathrm{mmoL}$ — milimoL; PEF — peak expiratory flow; Pre — present; Pred — predicted; R tot — total resistance; RV — residual volume; s — second; SB — single breath; TLC — total lung capacity; UL — upper line; VA — alveolar volume; VC — vital capacity; Vin — inspiratory volume

\section{Table 2. Results of bronchoalveolar lavage}

\begin{tabular}{|c|c|c|}
\hline Probe volume & & $115 \mathrm{~mL}$ \\
\hline Total cell count & \multicolumn{2}{|c|}{25.7 (non-smokers/smokers: $<10 \times 10^{6}<20 \times 10^{6}$ ) $\left[\times 10^{6}\right]$} \\
\hline Cellular viability & \multicolumn{2}{|r|}{$82[\%]$} \\
\hline macrophages & \multicolumn{2}{|c|}{84.7 (non-smokers/smokers: > 80\% > 90\%) [\%] } \\
\hline limphocytes & \multicolumn{2}{|c|}{9.6 (non-smokers/smokers: < 15\% < 10\%) [\%] } \\
\hline neutrophils & \multicolumn{2}{|c|}{4.8 (non-smokers/smokers: $<3 \%<3 \%$ ) [\%] } \\
\hline eozynophils & \multicolumn{2}{|c|}{0.9 (non-smokers/smokers: $<0,5 \%<0,5 \%$ ) [\%] } \\
\hline Probe description after MGG staining & \multicolumn{2}{|c|}{$\begin{array}{l}\text { Significant amount of foamy macrophages, single amount of macrophages with small vacuole, } \\
\text { single erythrocytes and epithelial cells }\end{array}$} \\
\hline \multicolumn{3}{|l|}{ MGG — May-Grünwald Giemsa } \\
\hline \multicolumn{3}{|c|}{$\begin{array}{ll}\begin{array}{l}\text { The exogenous type of lipoid pneumonia } \\
\text { occurs as a result of exposure to various fatty }\end{array} & \begin{array}{l}\text { tion-related syndromes in patients with impaired } \\
\text { substances (mostly mineral oils), which may be }\end{array} \\
\text { [5]. Rare cases of exogenous lipoid pneumonia are } \\
\text { inhaled or ingested. This form of disease con- } & \text { mentioned in the literature after aspiration during } \\
\text { cerns adults with chronic obstipation treated } & \text { 'fire-eating' or in patients after laryngectomy due } \\
\text { with oil-based laxatives, chronic rhinopharyngeal } & \text { to administration of mineral-oil containing pro- } \\
\text { diseases treated with nasal drops, or due to aspira- } & \text { ducts through the tracheostomy [3, } 4,6] .\end{array}$} \\
\hline
\end{tabular}


In our case report, we present an example of exogenous form of lipoid pneumonia, caused by chronic nasal drop use, in a patient with deviated nasal septum.

In the presented woman, the first suggestion concerning the fatty-like character of pulmonary opacities came from chest CT description. Similarly to our case, the most frequent radiologic manifestations described in the patients with lipoid pneumonia, are ground-glass opacities and consolidations involving one or more segments, typically with peribronchovascular distribution and predominant location in both lower lobes, with the adipose density (between -150 and -30 HU), which can be found in $70-80 \%$ of patients $[6,8]$. Other described forms are nodules and masses with irregular margins, which may be misdiagnosed as lung cancer [3,6].

The confirmation of diagnosis, next to the radiological finding, may be achieved by demonstration of lipid-laden macrophages in BAL, in a patient with the history of lipid exposure. To verify the diagnosis, staining specific for lipids is often necessary [3, 6, 8, 9]. If the triad of history of mineral-oil ingestion, imaging finding and BAL finding are not decisive, transthoracic fineneedle biopsy, transbronchial lung biopsy may be required. Surgical lung biopsy as the most invasive procedure should be reserved for the case of failure of other less invasive methods. Fine-needle biopsy or resection may be necessary if there is a risk of neoplasmatic process $[2,3,6$, 8]. In the presented case, the patient's history revealed an over 10 years long exposure to paraffin oil-containing nasal drops, which in conjunction with CT scan findings and the presence of foamy macrophages in BAL, confirmed the diagnosis of exogenous lipoid pneumonia.

Essential for treatment of lipoid pneumonia is to identify and discontinue exposure. Other treatments like steroids therapy or lung lavage are based on other case reports and should be reserved for severe forms of disease. Lipoid pneumonia is commonly indolent and may regress without treatment [3, 6, 8]. However, in some cases, the radiological changes may be improving over time despite the discontinuation of exposure. Progression may lead to fibrosis and subsequently result in pulmonary hypertension [3].
Our patient was advised to stop using nasal drops. Due to normal lung function parameters, no further therapy was indicated.

\section{Conclusions}

Lipoid pneumonia is an unusual cause of respiratory symptoms and can be either endogenous or exogenous. Clinical and radiological manifestations are diverse, therefore, in order to confirm the diagnosis of exogenous lipoid pneumonia, clinical history of lipid aspiration or inhalation, and proving the presence of lipid -ladden macrophages in respiratory specimen are mandatory.

\section{Conflict of interest}

The authors declare no conflict of interest.

\section{References:}

1. Betancourt SL, Martinez-Jimenez S, Rossi SE, et al. Lipoid pneumonia: spectrum of clinical and radiologic manifestations. AJR Am J Roentgenol. 2010; 194(1): 103-109, doi: 10.2214/AJR.09.3040, indexed in Pubmed: 20028911.

2. Krychniak-Soszka A, Lewandowska K, Skorupa W, et al. Egzogenne lipidowe zapalenie płuc- różnorodność obrazu klinicznego i radiologicznego. Pneumonol Alergol Pol. 2005; 73: 182-188.

3. Hadda V, Khilnani GC. Lipoid pneumonia: an overview. Expert Rev Respir Med. 2010; 4(6): 799-807, doi: 10.1586/ers.10.74, indexed in Pubmed: 21128754.

4. Załeska J, Ptaszek B, Malong P, et al. Lipoid pneumonia in patients after laryngectomy. Otolaryngol Pol. 2007; 61(6): 1004-1010, doi: 10.1016/S0030-6657(07)70571-1, indexed in Pubmed: 18546953.

5. Hu X, Lee JS, Pianosi PT, et al. Aspiration-related pulmonary syndromes. Chest. 2015; 147(3): 815-823, doi: 10.1378/ chest.14-1049, indexed in Pubmed: 25732447.

6. Marchiori E, Zanetti G, Mano CM, et al. Exogenous lipoid pneumonia. Clinical and radiological manifestations. Respir Med. 2011; 105(5): 659-666, doi: 10.1016/j.rmed.2010.12.001, indexed in Pubmed: 21185165.

7. Ramin S, Mohammadali Z, Forozan M. Adult onset still disease associated with endogenous lipoid pneumonia. ARM. 2019; 87(1): 50-53, doi: 10.5603/ARM.a2019.0008, indexed in Pubmed: 30830958.

8. Osman GA, Ricci A, Terzo F, et al. Exogenous lipoid pneumonia induced by nasal decongestant. Clin Respir J. 2018; 12(2): 524531, doi: 10.1111/crj.12557, indexed in Pubmed: 27717235.

9. Kim CHo, Kim EJ, Lim JK, et al. Comparison of exogenous and endogenous lipoid pneumonia: the relevance to bronchial anthracofibrosis. J Thorac Dis. 2018; 10(4): 2461-2466, doi: 10.21037/jtd.2018.04.06, indexed in Pubmed: 29850153.

10. Wu XJ, Li M, Zhan QY. [Exogenous lipid pneumonia with hyperpyrexia: a case report]. Beijing Da Xue Xue Bao Yi Xue Ban. 2018; 50(5): 921-923, indexed in Pubmed: 30337759. 